arterial pressure than IRRT patients at the end of the study periods (weighted mean difference 5.35, 95\% Cl 1.41-9.29), indicating potential superiority of the former treatment modality in hemodynamically unstable patients. CRRT patients had an increased risk of recurrent dialysis filter clotting compared with IRRT patients (RR 8.50, 95\% Cl 1.14-63.33).

Original article Rabindranath $\mathrm{K}$ et al. Intermittent versus continuous renal replacement therapy for acute renal failure in adults. Cochrane Database of Systematic Reviews 2007, Issue 3. Art. No.: CD003373.pub3. doi:10.1002/14651858. CD003773.pub3

\section{High initial tacrolimus dosage recommended in patients switched from ciclosporin}

Some kidney transplant recipients who receive initially adequate doses of ciclosporin fail to achieve therapeutic $2 \mathrm{~h}$ post-dose $\left(\mathrm{C}_{2}\right)$ levels of this calcineurin inhibitor. One option in such cases is to switch patients to tacrolimus, which has a different pharmacokinetic profile. As the absorption rate ratios of the two drugs are, however, almost identical, a Canadian group has investigated whether 'converted' patients require higher tacrolimus doses than do de novo tacrolimus patients.

House et al. retrospectively identified 15 adult recipients of a first renal transplant who were converted from ciclosporin to tacrolimus (plus mycophenolate mofetil and steroids). These patients had failed to reach target $\mathrm{C}_{2}$ levels of ciclosporin despite dose increases, and were switched to tacrolimus a mean 5.1 days after transplantation. This group was compared with a group of 14 control patients treated initially with tacrolimus. Initial tacrolimus dosages were similar in the conversion and de novo groups ( 0.18 and $0.17 \mathrm{mg} / \mathrm{kg} /$ day, respectively), but converted patients required higher daily doses to achieve target trough levels (0.25 vs $0.16 \mathrm{mg} / \mathrm{kg} /$ day, $P=0.016$; target $10-15 \mathrm{ng} / \mathrm{ml}$ ). At 3 weeks, maintenance tacrolimus doses remained significantly higher in low ciclosporin absorbers than in the control patients ( 0.22 vs $0.13 \mathrm{mg} / \mathrm{kg} / \mathrm{day} ; P=0.012)$. The rate of acute rejection tended to be higher in converted patients than in controls (33\% vs $21 \%$ ).

The authors suggest that patients who switch to tacrolimus because of failure to reach target $\mathrm{C}_{2}$ ciclosporin levels should receive a high initial dosage (e.g. $0.25 \mathrm{mg} / \mathrm{kg} /$ day) of this immunosuppressive agent.

Original article House AA et al. (2007) Apparent low absorbers of cyclosporine microemulsion have higher requirements for tacrolimus in renal transplantation. Clin Transplant 21: 518-522

\section{Body-weight-adjusted mycophenolate mofetil doses in Asian renal transplant recipients}

The efficacy and toxicity of mycophenolate mofetil (MMF) are concentration-dependent, yet a standard fixed dose of $2 \mathrm{~g} /$ day is currently recommended for maintenance immunosuppression in adult renal transplant recipients also receiving ciclosporin and corticosteroids. Studies of Asian patients have indicated that MMF doses below 2 g/day can be effective, possibly because of the typically lower total body weight of Asian populations relative to those from Europe or North America.

Yau et al. examined the steady-state pharmacokinetics of mycophenolic acid (MPA), the active metabolite of MMF, in 53 Asian adult renal transplant recipients. Median total body weight was $66.8 \mathrm{~kg}$ (range $33.1-108.1 \mathrm{~kg}$ ). Patients had received MMF $(0.5-2.0 \mathrm{~g} /$ day; $86.8 \%$ were receiving a dose $<2.0 \mathrm{~g} /$ day), ciclosporin and prednisolone for at least 3 months before recruitment. Exposure to MPA correlated significantly with body-weight-adjusted MMF dose ( $r^{2}=0.49, P<0.0005$; after omission of outliers). Regression analysis revealed that therapeutic levels of MPA exposure (area under the plasmaconcentration-time curve at steady state of 30-60 mg•h/l) could be achieved by a twice daily dose of MMF at $5-17 \mathrm{mg} / \mathrm{kg}$. Most patients had MPA exposure within the therapeutic range, despite receiving MMF doses of $<2 \mathrm{~g} /$ day.

The authors suggest that an MMF dose of $12 \mathrm{mg} / \mathrm{kg}$ twice daily could be used to target the middle of the therapeutic range in Asian renal transplant recipients, and potentially in other ethnic groups. Considerable interindividual variability in pharmacokinetics was observed, however, even after adjustment for body weight, indicating that dose adjustment of MMF on the basis of therapeutic drug monitoring of MPA would be required.

Original article Yau WP et al. (2007) Is a standard fixed dose of mycophenolate mofetil ideal for all patients? Nephrol Dial Transplant [doi:10.1093/ndt/gfm468] 